\title{
CONCEPCIONES DEL ALUMNADO Y CONOCIMIENTO ESCOLAR. UNA APROXIMACIÓN AL CONCEPTO DE LA CONTAMINACIÓN.
}

\author{
Autora: Olga María Moreno Fernández \\ Directora: Dra. Dña. Macarena Esteban Ibáñez \\ Universidad:Tesis de maestría en Educación Ambiental. Universidad Pablo de Olavide (Sevilla) \\ Fecha de defensa: 4 de noviembre de 2010
}

\section{INTRODUCCIÓN}

Este trabajo de investigación se centra en el conocimiento de las ideas previas del alumnado y, en concreto, dentro del ámbito de la Educación Ambiental en el tema de la contaminación. Para su realización se partió de la elaboración y aplicación de un cuestionario sobre conocimientos e intereses hacia el problema que presenta la contaminación en distintos centros de Primaria en dos entornos urbanos. En el trabajo se analizan los resultados para cada una de las cuestiones planteadas y se comparan en función de la zona de ubicación de dichos centros. Finalmente se proponen una serie de sugerencias metodológicas para abordar la cuestión a tratar.

\section{OBJETIVOS GENERALES DEL TRABAJO DE INVESTIGACIÓN}

- Evaluar los conocimientos previos sobre la contaminación que tienen el alumnado del último curso del segundo ciclo de Primaria (4. ${ }^{\circ}$ E. P.) y el primer curso del tercer ciclo de Primaria (5. ${ }^{\circ}$ E. P.).

- Detectar las concepciones erróneas.

- Ver si existen diferencias entre los conocimientos de los escolares de Sevilla capital y la ciudad de Dos Hermanas, situándonos en dos contextos socio-económico-culturales totalmente opuestos.

- Ver las posibles aplicaciones y necesidades para hacer más eficaz el conocimiento y la sensibilización de los escolares con respecto al tema que nos ocupa.

\section{METODOLOGÍA}

La metodología con la que se ha abordado la investigación es de tipo cuantitativo, y la finalidad de la investigación es la de llegar a comprender cómo el alumnado genera sus conocimientos, si estos corresponden con lo ofertado desde las distintas programaciones de aula y desde los diversos materiales didácticos que las editoriales educativas ofertan, y qué sería necesario para que se llevara a cabo un aprendizaje significativo que cambie los esquemas de conocimientos del alumnado.

\section{RESULTADOS}

A través de los datos obtenidos de los cuestionarios podemos llegar a las siguientes conclusiones:

La mayor parte de nuestro alumnado entiende la contaminación como el acto de arrojar basura al suelo (36\%) o como el hecho de utilizar vehículos (20\%), aunque cabe resaltar como dato interesante que el 17,4 $\%$ del alumnado confunde la contaminación con otros tipos de problemas medioambientales tales como los incendios o el no reciclar. En lo que hemos podido observar mayor confusión es en lo que es la contaminación acústica, ya que nos encontramos con un gran número de discentes que no sabe lo que es (50\%).

El alumnado conoce en líneas generales que es la contaminación pero que desconoce los diferentes tipos de contaminación existentes reduciéndolas en la mayoría de los casos a la contaminación atmosférica, en los casos que se presentan otros tipos de contaminación los conceptos no los tienen claros.

En cuanto a la relación de centros y de cursos escolares podemos afirmar que la diferencia entre los conocimientos existentes en ambos centros es mínima, por lo que la hipótesis en la que se planteaba que la localización geográfica es un factor que influye en los conocimientos e intereses del alumnado en este caso concreto no se cumple, por lo que podemos decir que en este caso y aunque los centros se ubican en dos contextos totalmente opuestos, no es relevante.

En el análisis comparado de las programaciones de aula que se han tomado como guía, desde el primer curso de Primaria y hasta quinto de Primaria se puede observar que en ellas se trabaja o se hace referencia tanto a la contaminación atmosférica, como a la acústica y a la del agua, como a otros problemas medioambientales, aunque hay que señalar que estos contenidos son parciales y fragmentarios. 


\section{CONCLUSIONES}

De acuerdo con los resultados obtenidos y tras una valoración de las concepciones que se han recogido y analizado, podemos concluir reflexionando sobre el elevado número de discentes que entienden el concepto de contaminación de forma simple y parcial limitándolo a hechos como el de arrojar basura al suelo o el uso de vehículos de motor, que por otra parte también son los que están más presente en el entorno de sus vidas cotidianas. Esto nos lleva a tener presente que la importancia que radica en conocer las ideas previas del alumnado no sólo se encuentra en el conocimiento de la distorsión que puede presentar el pensamiento de los discentes, sino que permite ampliar las posibilidades de actuación del docente a la hora de diseñar estrategias que favorezcan el aprendizaje significativo.

\title{
DISEÑO, APLICACIÓN Y EVALUACIÓN DE UNA PROPUESTA DIDÁCTICA PARA TRABAJAR LA ENSEÑANZA DE LOS CIRCUITOS ELÉCTRICOS EN TERCER CICLO DE EDUCACIÓN PRIMARIA
}

\author{
Autor: Javier Rodríguez Moreno \\ Tipo de Tesis: Maestría \\ Director: Dr.Antonio de Pro Bueno \\ Departamento de Didáctica de las Ciencias Experimentales \\ Universidad de Murcia \\ Programa: Master Oficial en Investigación e Innovación en Educación Infantil y Educación Primaria \\ Fecha de presentación: junio de 2008
}

El objetivo central del trabajo fue diseñar, aplicar y evaluar la unidad didáctica "Estudio de los Circuitos Eléctricos en la Educación Primaria”. Por ello, articulamos la investigación para dar respuesta a los siguientes interrogantes:

P.P.1: ¿Cuáles eran los conocimientos iniciales que tenía el alumnado sobre los circuitos eléctricos? ¿Cómo considerarlos en la planificación de la propuesta?

P.P.2: ¿Cómo se desarrollaron las actividades previstas? ¿Qué logros se obtuvieron, qué dificultades se encontraron... en las actividades prácticas?

P.P.3: ¿Qué efectos produjo dicha propuesta en el alumnado en relación con el aprendizaje?

En primer lugar, revisamos qué decía el currículo respecto a estos contenidos y analizamos algunas contribuciones sobre la enseñanza y el aprendizaje de esta temática. En base a esta revisión y nuestra experiencia, establecimos las hipótesis a contrastar.

Respecto al diseño de la investigación, se trata del ensayo de una propuesta con pretest, seguimiento y postest. Los participantes correspondían a una clase de
5. ${ }^{\circ}$ de EP de un CEIP de una población de Andalucía; se identificaron características personales y académicas que delimitaban el contexto de la experiencia.

Para la planificación de la Unidad Didáctica, utilizamos un modelo basado en la realización de cinco tareas: análisis del contenido científico, análisis de los problemas de aprendizaje, determinación de los objetivos de aprendizaje, selección de estrategias de enseñanza y selección de estrategias de evaluación.

Para valorarla, utilizamos un pretest y un postest; en ambos casos, se trataba de cuestionarios de cuestiones cotidianas. En relación con el seguimiento, hicimos una valoración de cada sesión mediante el diario del maestro; además, nos centramos en las tres actividades prácticas (estudio de circuitos con bombillas simples, en serie y en paralelo). En todos los casos, nos fijamos de forma especial en la presencia de los modelos alternativos de corriente (fluido, unipolar, interruptor-fuente, atenuación...).

Respecto a los resultados del Problema Principal Uno, podemos destacar que, a pesar de que el alumnado no había trabajado estos conocimientos en esta etapa, sus experiencias les permitían distinguir aparatos eléctricos de su entorno, conocer algunas de las 
normas de seguridad o identificar los elementos de un circuito sencillo. Esto puso de manifiesto una vez más que los niños de estas edades no son una "tabla rasa" a la hora de construir los conocimientos. De hecho, no sólo los consideramos en el enfoque de los materiales sino que utilizamos sus ideas continuamente en el propio proceso de construcción de sus aprendizajes. No obstante, también detectamos que no sentían, en general, la necesidad de utilizar un modelo de corriente en sus explicaciones y justificaciones.

Respecto a los resultados del Problema Principal Dos, el desarrollo de la propuesta se ajustó a lo previsto en la planificación. El alumnado se implicó desde el principio y creemos que disfrutó aprendiendo. El trabajo en grupo favoreció el aprendizaje; probablemente porque era habitual en la clase. La incorporación de prácticas de laboratorio no sólo les estimuló sino que incrementó su interés. Resultó muy interesante el uso de los cuadernos de trabajo, en los que se recogían las preguntas que les planteábamos, sus respuestas y sus discusiones en grupo; en este sentido, se pudo observar una mejora sustantiva en la comunicación escrita. También nosotros disfrutamos a lo largo de nuestra experiencia.

En cuanto al seguimiento de algunas actividades prácticas, hemos de señalar que, en la referida al circuito simple, todos realizaron el montaje sin aparentes dificultades, comprobaron su funcionamiento e, incluso, no tuvieron problemas en la descripción e interpretación de lo realizado. En la práctica del circuito con bombillas en serie, también todos realizaron el montaje sin dificultad y contestaron adecuadamente las preguntas referidas a la descripción de lo que iban observando; sin embargo, se apreciaron dificultades cuando debían explicar, interpretar, predecir... hechos y fenómenos que habían realizado o provocado. En el circuito con bombillas en paralelo tuvimos peores resultados porque algunos tuvieron problemas en la realización del montaje; este hecho acrecentó las limitaciones en las cuestiones de interpretación, predicción y aplicación de los resultados.

Respecto a los resultados en el Problema Principal Tres, el alumnado mantuvo los logros iniciales y mejoraron las respuestas en relación con la interpretación del funcionamiento de los circuitos. No obstante, aparecieron en algunos casos y mantuvieron en otros los modelos alternativos de corriente eléctrica; estos condicionaron muchos de los razonamientos realizados. En cualquier caso, creemos que el interés y la motivación que se alcanzaron podrían ir más allá de los efectos en el aprendizaje a corto plazo que hemos detectado.

Globalmente nos parece una experiencia muy útil e interesante para la mejora de nuestro ejercicio profesional. Desde nuestro punto de vista, para una adecuada evolución y superación de los problemas que hemos debido afrontar, creemos necesario seguir investigando sobre las propuestas de enseñanza que realizamos todos los días en esta y otras etapas educativas.

\title{
LOS CONCEPTOS DE POBLACIÓN Y DE ESPECIE EN LA ENSEÑANZA DE LA BIOLOGÍA: CONCEPCIONES, DIFICULTADES Y PERSPECTIVAS.
}

\author{
Autora: M. ${ }^{a}$ del Pilar Jiménez Tejada \\ Tipo:Tesis doctoral. \\ Directores: Francisco González García y José A. Hódar Correa. \\ Departamento: Didáctica de las Ciencias Experimentales. \\ Universidad de Granada. \\ Programa de Doctorado de Didáctica de las Ciencias Experimentales. \\ Fecha de presentación: 5 de marzo de 2009
}

El interés de los conceptos de población y especie, evidenciado por los numerosos debates que suscitan entre los científicos, no es equivalente a las atenciones recibidas desde la investigación en didáctica de la biología. La importancia de ambos términos radica en su relación con la teoría de la evolución. También vertebran otras áreas de conocimiento en que se divide la biología, como la genética, la ecología, todas las 
que abordan la taxonomía o la moderna biología de la conservación.

En esta investigación se han puesto de manifiesto las ideas alternativas presentes en el alumnado universitario. Para ello se recogieron 615 encuestas estructuradas entre estudiantes universitarios de biología y ciencias ambientales. En el cuestionario debían diferenciar dichos términos y aplicarlos a un caso práctico.

El análisis de las encuestas mostró diferencias significativas entre el alumnado de las diversas licenciaturas universitarias, y aparece un porcentaje más alto de respuestas correctas en los grupos de biología. No obstante, el porcentaje de respuestas correctas no ha sido superior en el alumnado de cursos más elevados de biología. También se apreciaron frecuentes confusiones entre los conceptos de especie y reino, población y comunidad, incluso en estudiantes de últimos cursos.

Se ha observado que el pensamiento tipológico está muy extendido en los estudiantes de todas las licenciaturas, incluso en los de biología de cursos superiores.

La aplicación de ambos términos a una situación concreta también estuvo influida por la instrucción recibida, y existe una tendencia a presentarse mejores respuestas en cursos superiores, pero no en el último curso.

La persistencia o cambio de las ideas alternativas se ve influida por las vivencias de los alumnos, pero también por la formación recibida a su paso por las enseñanzas medias y durante sus estudios universitarios. Por ello, como segunda gran tarea, hemos analizado el tratamiento que el profesorado universitario y de enseñanzas medias da a los conceptos de población y especie, y los libros de texto y los manuales especializados de ecología y genética.

En la revisión de 19 textos de ecología y 13 de genética, analizamos cómo y dónde aparecían definidos los conceptos de población y especie. Cabría esperar que las definiciones apareciesen en capítulos estrechamente relacionados o, al menos, en glosario. Sin embargo, algunos textos no las incluían en ninguna de las dos partes.

La forma de presentar las definiciones de ambos conceptos debería ser más cuidadosa, huyendo de términos que puedan contribuir a la confusión del alumnado (como el de comunidad en algunos textos de genética) o completándolas para cubrir aspectos necesarios para diferenciarlas de otros términos.

De los cuestionarios recogidos entre el profesorado universitario no se puede extraer mucha información, dado el escaso número de personas que participaron, diez en total; aunque si podemos percibir el escaso interés que despierta la investigación en didáctica entre este profesorado, una dificultad más para la mejora en su docencia.

La revisión de textos de enseñanza media relacionados con la biología muestran resultados similares a los encontrados en los manuales especializados, y presentan algunas carencias en las definiciones u ofreciendo visiones parciales de ambos conceptos. Las definiciones de especie tienden a mostrar una visión tipológica del concepto.

El profesorado entrevistado, un total de 67, contestaron un cuestionario sobre sus estrategias de enseñanza. Los profesores no aprecian grandes dificultades en la comprensión de dichos conceptos por parte de los alumnos, lo que podría condicionar los procedimientos empleados en su enseñanza.

Las diferencias existentes entre los dos conceptos, su definición en todos los temas relacionados con ellos, o el uso de actividades y ejemplos, son prácticas habituales que están bastante extendidas entre el profesorado, aunque existe un pequeño porcentaje que no siempre las utilizan. El uso de lecturas complementarias o las salidas al campo, que podrían mejorar el aprendizaje y la motivación del alumnado, son escasamente utilizadas.

El concepto de población se puede trabajar de forma interdisciplinar desde las matemáticas y las ciencias sociales por lo que también se ha investigado cómo se plantea dicho concepto desde ambas disciplinas, tanto en los textos como por el profesorado. Con esta investigación comprobamos los nexos con esas disciplinas y sugerimos propuestas para la mejora de la enseñanzaaprendizaje de estos conceptos. La interpretación, elaboración y el estudio comparativo de las pirámides de poblaciones y las gráficas de crecimiento poblacional de la especie humana y de otras especies, ayudaría a despojarnos de la visión antropocéntrica tan arraigada en nuestro pensamiento. 